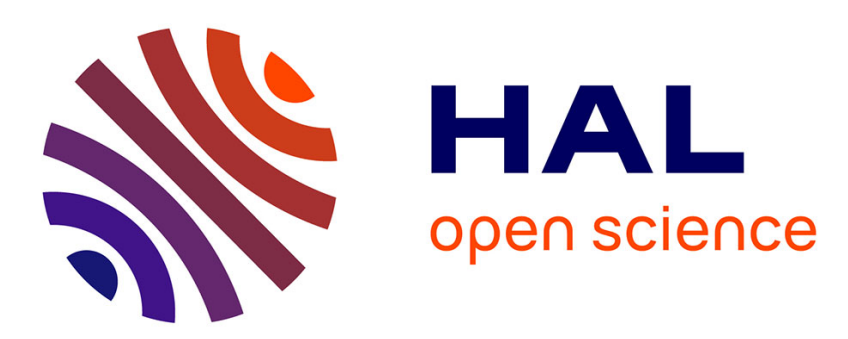

\title{
Adaptive backstepping for trajectory tracking of nonlinearly parameterized class of nonlinear systems
}

Hakim Bouadi, Felix Mora-Camino

\section{To cite this version:}

Hakim Bouadi, Felix Mora-Camino. Adaptive backstepping for trajectory tracking of nonlinearly parameterized class of nonlinear systems. CINTI 2011 IEEE 12th International Symposium on Computational Intelligence and Informatics, Nov 2011, Budapest, Hungary. pp 213 - 217, 10.1109/CINTI.2011.6108501 . hal-00938519

\section{HAL Id: hal-00938519 \\ https://hal-enac.archives-ouvertes.fr/hal-00938519}

Submitted on 20 Jun 2014

HAL is a multi-disciplinary open access archive for the deposit and dissemination of scientific research documents, whether they are published or not. The documents may come from teaching and research institutions in France or abroad, or from public or private research centers.
L'archive ouverte pluridisciplinaire HAL, est destinée au dépôt et à la diffusion de documents scientifiques de niveau recherche, publiés ou non, émanant des établissements d'enseignement et de recherche français ou étrangers, des laboratoires publics ou privés. 


\title{
Adaptive Backstepping for Trajectory Tracking of Nonlinearly Parameterized Class of Nonlinear Systems
}

\author{
Hakim Bouadi, F. Mora-Camino
}

\begin{abstract}
Adaptive control is of interest in control systems design not only for its capability to improve performance and reliability but also for handling parameters uncertainties, external disturbances and modeling inaccuracies. This paper addresses a systematic procedure design to develop an adaptive backstepping tracking control approach for a second-order class of single input-single output (SISO) nonlinear systems where the controllability condition is checked. The considered class covers general feedback linearizable and nonlinearly parameterized systems. Both of the control and adaptation laws are synthesized simultaneously based on the positivity and Lyapunov design approaches. Simulation results for an illustrative numerical example are provided to illustrate in this case the effectiveness of the proposed controller.
\end{abstract}

Index Terms-adaptive backstepping, adaptation law, positivity principle.

\section{INTRODUCTION}

$\mathbf{N}$ ONLINEAR adaptive control has seen a growing interest in the past two decades. Several approaches are proposed in the literature considering various classes of nonlinear systems. There are at least two main commun factors between the considered classes. The proposed approaches cover both class of general feedback linearizable systems and linearly parameterized one.

Nonlinear adaptive control for linearly parameterized nonlinear systems has been one of the active subjects in the field of nonlinear control [1], [2], [3], [4] and [5] while lot of dynamic processes from chemical industry and biotechnology are characterized by nonlinearly parameterized models such as distillation columns, chemical reactors, friction dynamics, bioreactors and flight dynamics. In fact, several researchers started working on this difficult problem and obtained some interesting results [9]-[12]. It must be noticed that most of the results are derived under various conditions imposed on the parameters such as convex or concave parameterization [13].

Among proposed solutions to overcome the problem of nonlinear parameterization is to find a transformation which

H. Bouadi is PhD student and he is with the Automation and Operational Research Laboratory (LARA) in the National Civil Aviation InstituteENAC. 07, Avenue Edouard Belin, 31400-Toulouse-France (phone: +33 (0)-6 01-18-83-91, fax: +33 (0)-5-62-17-40-23 hakimbouadieyahoo. fr, Hakim. Bouadi@enac. fr.

F. Mora-Camino is Professor at the National Civil Aviation Institute and he is the director of the Automation and Operational Research Laboratory (LARA) at Air Transportation Department. moracamino@hotmail.fr.

Manuscript received September 29, 2011. This work was supported by Automation and Operational Research Laboratory of Air Transportation Department in the National Civil Aviation Institute in Toulouse-France. transforms nonlinearly parameterized systems into systems with linear parameterization. However, this task remains sometimes very difficult to perform and there is no systematic method available currently [12].

On the other hand, adaptive control for some classes of nonlinearly parameterized systems has been attempted in [6], [7], [8], [9], [14] and [15]. The approach used in [8] deals with the problem of adaptive regulation of a class of nonlinear systems. Authors in [7] solve the tracking problem in the case of first-order plants containing the simplest possible nonlinearity encountered in bioreactor process models. In [9] has developed a method for adaptive control design for a class of first-order systems containing nonlinearities of the form of ratios of polynomials in the process output with unknown parameters.

In [13] a systematic design procedure is given for the explicit construction of one dimensional, smooth adaptive controllers which achieve asymptotic state regulation with global stability. As a consequence, global adaptive stabilization of feedback linearizable and nonlinearly parameterized systems is solvable using a smooth, one dimensional adaptive controller, without requiring any condition such as convex/concave condition [12] on the unknown parameters.

During the last few years, controllers based-on the backstepping approach have emerged as powerful tool for stabilizing nonlinear systems both for tracking and regulation problems [1]. The main advantage of this approach is the systematic construction of a Lyapunov positive definite function for the closed-loop, allowing the analysis of its stability propertiers.

For the adaptive version, in [10], for triangular systems, robust adaptive control techniques are incorporated in the backstepping control design with flat zones to tackle the nonlinear parameterization together with a novel smooth projection algorithms for parameter estimation. For a class of nonlinearly parameterized cascade systems, a solution to the problem of global adaptive regulation is given in [11] by using a novel parameter separation technique combined with a feedback domination design [16]. By comparaison, only few results for Adaptive backstepping tracking control for nonlinearly parameterized nonlinear systems have been established.

In this paper, an adaptive backstepping systematic procedure tracking control design for nonlinearly parameterized class of second-order nonlinear systems is developed without requiring any restriction on the unknown parameter. Two main advantages are verified for the considered class, the controllablity condition and the non-existence of internal dynamics 
for the considered output. Both of the control and adaptation laws are synthesized simultaneously based on the Lyapunov design approach.

To test the practical effectiveness of the proposed controller design, developed approach has been applied to a numerical illustrative example. Simulation results in this case show satisfactory performances in term of tracking and estimation, respectively.

\section{Problem Formulation}

\section{A. Preliminaries}

Consider a class of nonlinear systems:

$$
\begin{gathered}
\dot{x_{1}}=x_{2}+\theta f\left(x_{1}, \theta\right) \\
\dot{x_{2}}=u \\
y=x_{1}
\end{gathered}
$$

where $f$ is a known smooth nonlinear differentiable function according to $x_{1}$ and $\theta$, respectively. $\theta \in \mathbb{R}$ represents an unknown parameter to be estimated. $x \in \mathbb{R}^{2}$ denotes the state vector, $u \in \mathbb{R}$ represents the control input and $y \in \mathbb{R}$ is the considered output.

The main objective of this paper is to highlight a systematic procedure design for the development of a controller based-on an adaptive backstepping approach in order to track a desired trajectory $x_{1 d}$ and to estimate the unknown parameter $\theta$ for the SISO considered class of nonlinear systems.

The relative degree of the considered output $y$ is equal to two since the control input $u$ appears in its second time derivative. Then no internal dynamics is associated with this output.

Considered class can be written under the nonlinear affine state representation :

$$
\dot{x}=h(x)+g(x) u
$$

where:

$$
h(x)=\left(\begin{array}{c}
x_{2}+\theta f\left(x_{1}, \theta\right) \\
0
\end{array}\right), g(x)=\left(\begin{array}{l}
0 \\
1
\end{array}\right)
$$

The nonlinear system (2) is input-state linearizable if, and only if, there exist a region $\Omega \subset \mathbb{R}^{n}$ such that the vector fields $\left[g, a d_{h} g, a d_{h}^{2} g, \ldots . ., a d_{h}^{n-1} g\right]$ are linearly independent in $\Omega$ and the set $\left[g, a d_{h} g, a d_{h}^{2} g, \ldots . ., a d_{h}^{n-2} g\right]$ is involutive in $\Omega$ [17]. Note that the first condition check the controllability of the system where $n$ denotes the system order and $a d_{h} g$ represents the Lie brackets such as:

$$
a d_{h} g=\nabla g h-\nabla h g
$$

The controllability matrix $\Upsilon$ for the system (2) given by:

$$
\Upsilon=\left[g, a d_{h} g\right]
$$

is such that:

$$
\Upsilon=\left(\begin{array}{cc}
0 & -1 \\
1 & 0
\end{array}\right)
$$

and

$$
\operatorname{det}(\Upsilon)=1
$$

since $\operatorname{det}(\Upsilon) \neq 0$, it can be concluded that $\forall\left(x_{1}, x_{2}\right) \in \mathbb{R}^{2}$ and $\forall f\left(x_{1}, \theta\right) \in \mathbb{R}$ the considered above system is locally controllable over $\mathbb{R}^{2}$.

\section{B. Control Design}

To synthesize a control law $u$ based on an adaptive backstepping approach for the considered class of nonlinear systems, let us consider the state variable $x_{2}$ as a virtual control input for $x_{1}$ and the tracking error $\tilde{x}_{1}$ such as:

$$
\tilde{x}_{1}=x_{1}-x_{1 d}
$$

Now, let us introduce a candidate Lyapunov positive definite function $V_{1}\left(\tilde{x}_{1}\right)$

$$
V_{1}\left(\tilde{x}_{1}\right)=\frac{1}{2} \tilde{x}_{1}^{2}
$$

and the time derivative of the considered Lyapunov function is:

$$
\begin{aligned}
\frac{d V_{1}\left(\tilde{x}_{1}\right)}{d t} & =\tilde{x}_{1} \dot{\tilde{x}}_{1} \\
& =\tilde{x}_{1}\left[x_{2}+\theta f\left(x_{1}, \theta\right)-\dot{x}_{1 d}\right]
\end{aligned}
$$

The asymptotic Lyapunov stability is guaranteed if $\dot{V}_{1}\left(\tilde{x}_{1}\right)<0$. For that, the virtual control input $x_{2}$ can be chosen as:

$$
x_{2}=-k_{1} \tilde{x}_{1}-\theta f\left(x_{1}, \theta\right)+\dot{x}_{1 d}
$$

where $k_{1}$ is a real positive parameter. Since $\theta$ is an unknown parameter, it is replaced by its estimate $\hat{\theta}$ and $x_{2}$ becomes:

$$
x_{2}=-k_{1} \tilde{x}_{1}-\hat{\theta} f\left(x_{1}, \hat{\theta}\right)+\dot{x}_{1 d}
$$

Let $\xi$ be an auxiliary tracking error variable such as:

$$
\xi=x_{2}+k_{1} \tilde{x}_{1}+\hat{\theta} f\left(x_{1}, \hat{\theta}\right)-\dot{x}_{1 d}
$$

since the time derivative of the tracking error $\tilde{x}_{1}$ is:

$$
\dot{\tilde{x}}_{1}=x_{2}+\theta f\left(x_{1}, \theta\right)-\dot{x}_{1 d}
$$

and by replacing $x_{2}$ by its expression from (13), we get:

$$
\dot{\tilde{x}}_{1}=-k_{1} \tilde{x}_{1}+\xi+\hat{\theta}\left[f\left(x_{1}, \theta\right)-f\left(x_{1}, \hat{\theta}\right)\right]+\tilde{\theta} f\left(x_{1}, \theta\right)
$$

where $\tilde{\theta}$ represents the estimation error:

$$
\tilde{\theta}=\theta-\hat{\theta}
$$

In the steady state and by choosing an adequate gain adaptation, it can be written that the time derivative of the tracking error $\tilde{x}_{1}$ in (15) becomes:

$$
\dot{\tilde{x}}_{1}=-k_{1} \tilde{x}_{1}+\xi+\tilde{\theta} f\left(x_{1}, \hat{\theta}\right)
$$

where:

$$
\lim _{t \rightarrow \infty} \hat{\theta}(t)=\theta
$$


From (13), the time derivative of the auxiliary tracking error variable $\xi$ is:

$$
\begin{aligned}
\dot{\xi}= & u+\dot{\hat{\theta}} f\left(x_{1}, \hat{\theta}\right)+\hat{\theta}\left[\frac{\partial f\left(x_{1}, \hat{\theta}\right)}{\partial x_{1}} \dot{x}_{1}+\frac{\partial f\left(x_{1}, \hat{\theta}\right)}{\partial \hat{\theta}} \dot{\hat{\theta}}\right] \\
& -\ddot{x}_{1 d}+k_{1}\left[-k_{1} \tilde{x}_{1}+\xi+\tilde{\theta} f\left(x_{1}, \hat{\theta}\right)\right] \\
= & u+\dot{\hat{\theta}}\left[f\left(x_{1}, \hat{\theta}\right)+\hat{\theta} \frac{\partial f\left(x_{1}, \hat{\theta}\right)}{\partial \hat{\theta}}\right]-\ddot{x}_{1 d}-k_{1}^{2} \tilde{x}_{1}+k_{1} \xi \\
& +\hat{\theta} \frac{\partial f\left(x_{1}, \hat{\theta}\right)}{\partial x_{1}}\left[x_{2}+\hat{\theta} f\left(x_{1}, \theta\right)\right] \\
& +\tilde{\theta}\left[k_{1} f\left(x_{1}, \hat{\theta}\right)+\hat{\theta} \frac{\partial f\left(x_{1}, \hat{\theta}\right)}{\partial x_{1}} f\left(x_{1}, \theta\right)\right]
\end{aligned}
$$

and by replacing the unknown parameter $\theta$ by its estimate $\hat{\theta}$, we get:

$$
\begin{aligned}
\dot{\xi}= & u+\dot{\hat{\theta}} \phi_{1}\left(x_{1}, \hat{\theta}\right)+\hat{\theta} \phi_{2}(x, \hat{\theta})+\tilde{\theta} \phi_{3}\left(x_{1}, \hat{\theta}\right) \\
& -\ddot{x}_{1 d}-k_{1}^{2} \tilde{x}_{1}+k_{1} \xi
\end{aligned}
$$

where:

$$
\begin{array}{r}
\phi_{1}\left(x_{1}, \hat{\theta}\right)=f\left(x_{1}, \hat{\theta}\right)+\hat{\theta} \frac{\partial f\left(x_{1}, \hat{\theta}\right)}{\partial \hat{\theta}} \\
\phi_{2}(x, \hat{\theta})=\frac{\partial f\left(x_{1}, \hat{\theta}\right)}{\partial x_{1}}\left[x_{2}+\hat{\theta} f\left(x_{1}, \hat{\theta}\right)\right] \\
\phi_{3}\left(x_{1}, \hat{\theta}\right)=k_{1} f\left(x_{1}, \hat{\theta}\right)+\hat{\theta} \frac{\partial f\left(x_{1}, \hat{\theta}\right)}{\partial x_{1}} f\left(x_{1}, \hat{\theta}\right)
\end{array}
$$

In order to synthesize both the control law and the adaptation mechanism simultaneously, another Lyapunov positive definite function $V\left(\tilde{x}_{1}, \xi, \tilde{\theta}\right)$ is introduced such as:

$$
V\left(\tilde{x}_{1}, \xi, \tilde{\theta}\right)=V_{1}\left(\tilde{x}_{1}\right)+\frac{1}{2}\left(\xi^{2}+\frac{1}{\gamma} \tilde{\theta}^{2}\right)
$$

where $\gamma>0$ denotes the gain adaptation.

$$
\begin{aligned}
\frac{d V\left(\tilde{x}_{1}, \xi, \tilde{\theta}\right)}{d t}= & \tilde{x}_{1} \dot{\tilde{x}}_{1}+\xi \dot{\xi}-\frac{1}{\gamma} \tilde{\theta} \dot{\hat{\theta}} \\
= & -k_{1} \tilde{x}_{1}^{2}+\tilde{x}_{1} \xi+\tilde{\theta}\left[\tilde{x}_{1} f\left(x_{1}, \hat{\theta}\right)+\xi \phi_{3}\left(x_{1}, \hat{\theta}\right)\right. \\
& \left.-\frac{\dot{\hat{\theta}}}{\gamma}\right]+\xi\left[u+\dot{\hat{\theta}} \phi_{1}\left(x_{1}, \hat{\theta}\right)+\hat{\theta} \phi_{2}(x, \hat{\theta})\right. \\
& \left.-\ddot{x}_{1 d}-k_{1}^{2} \tilde{x}_{1}+k_{1} \xi\right]
\end{aligned}
$$

The control law $u$ and the adaptation mechanism $\dot{\hat{\theta}}$ can be chosen as:

$$
\begin{gathered}
u=-\dot{\hat{\theta}} \phi_{1}\left(x_{1}, \hat{\theta}\right)-\hat{\theta} \phi_{2}(x, \hat{\theta})+\ddot{x}_{1 d}+\left(k_{1}^{2}-1\right) \tilde{x}_{1} \\
-\left(k_{1}+k_{2}\right) \xi \\
\dot{\hat{\theta}}=\gamma\left[\tilde{x}_{1} f\left(x_{1}, \hat{\theta}\right)+\xi \phi_{3}\left(x_{1}, \hat{\theta}\right)\right]
\end{gathered}
$$

where $k_{2}$ is a real positive parameter. For that, the asymptotic Lyapunov stability is guaranteed since:

$$
\dot{V}\left(\tilde{x}_{1}, \xi, \tilde{\theta}\right)=-k_{1} \tilde{x}_{1}^{2}-k_{2} \xi^{2} \leqslant 0
$$

\section{ILlustrative Numerical EXAMPLe}

Let us consider the following system:

$$
\begin{gathered}
\dot{x_{1}}=x_{2}+\theta\left[x_{1}^{2}+\sin \left(\theta x_{1}\right)\right] \\
\dot{x_{2}}=u \\
y=x_{1}
\end{gathered}
$$

where the nonlinear function $f\left(x_{1}, \theta\right)$ which contains the unknown parameter $\theta$ is:

$$
f\left(x_{1}, \theta\right)=x_{1}^{2}+\sin \left(\theta x_{1}\right)
$$

From (5), it can be seen that the rank of the controllability matrix $\Upsilon$ of (26) is equal to two, so the considered system is locally controllable over $\mathbb{R}^{2}, \forall\left(x_{1}, x_{2}\right) \in \mathbb{R}^{2}$ and $\forall f\left(x_{1}, \theta\right) \in$ $\mathbb{R}$.

By referring to the previous section, the control law is as follows:

$$
\begin{aligned}
u= & -\dot{\hat{\theta}} \phi_{1}\left(x_{1}, \hat{\theta}\right)-\hat{\theta} \phi_{2}(x, \hat{\theta})+\ddot{x}_{1 d}+\left(k_{1}^{2}-1\right) \tilde{x}_{1} \\
& -\left(k_{1}+k_{2}\right) \xi
\end{aligned}
$$

with:

$$
\begin{gathered}
\phi_{1}\left(x_{1}, \hat{\theta}\right)=f\left(x_{1}, \hat{\theta}\right)+\hat{\theta} x_{1} \cos \left(\hat{\theta} x_{1}\right) \\
\phi_{2}(x, \hat{\theta})=\hat{\theta} f\left(x_{1}, \hat{\theta}\right)\left[2 x_{1}+\hat{\theta} \cos \left(\hat{\theta} x_{1}\right)\right]+2 x_{1} x_{2} \\
+\hat{\theta} x_{2} \cos \left(\hat{\theta} x_{1}\right)
\end{gathered}
$$

and the adaptation law is:

$$
\begin{aligned}
\dot{\hat{\theta}} & =\gamma\left[\tilde{x}_{1} f\left(x_{1}, \hat{\theta}\right)+\xi \phi_{3}\left(x_{1}, \hat{\theta}\right)\right] \\
& =\gamma f\left(x_{1}, \hat{\theta}\right)\left[\hat{\theta}^{2} \xi \cos \left(\hat{\theta} x_{1}\right)+2 \hat{\theta} \xi x_{1}+k_{1} \xi+\tilde{x}_{1}\right]
\end{aligned}
$$

with:

$$
\phi_{3}\left(x_{1}, \hat{\theta}\right)=f\left(x_{1}, \hat{\theta}\right)\left[k_{1}+\hat{\theta}\left[2 x_{1}+\hat{\theta} \cos \left(\hat{\theta} x_{1}\right)\right]\right]
$$

\section{Simulation Results}

For the application of the numerical example developed above, two cases are considered. The first one concerns the estimation of the unknown parameter $\theta$ which is taken as a constant while in the second case, $\theta$ is taken as a nonlinear function of time. Both of the two considered cases are subject of two studies. Firstly, the simulations are performed without noise. After, a white gaussian noise $w(0, \sigma)$ with various variances is added to the output $y=x_{1}$ such as:

$$
\begin{gathered}
\sigma_{w_{1}}=125 \times 10^{-7} \\
\sigma_{w_{2}}=125 \times 10^{-4} \\
\sigma_{w_{3}}=0.25
\end{gathered}
$$

where $\sigma$ denotes the variance of the considered white gaussian noise. For the first case, the simulation data are: $x_{1}(0)=$ $0, \gamma=0.35$ and $\hat{\theta}(0)=2.5$ where the parameter $\theta$ to be estimated is:

$$
\theta=2
$$



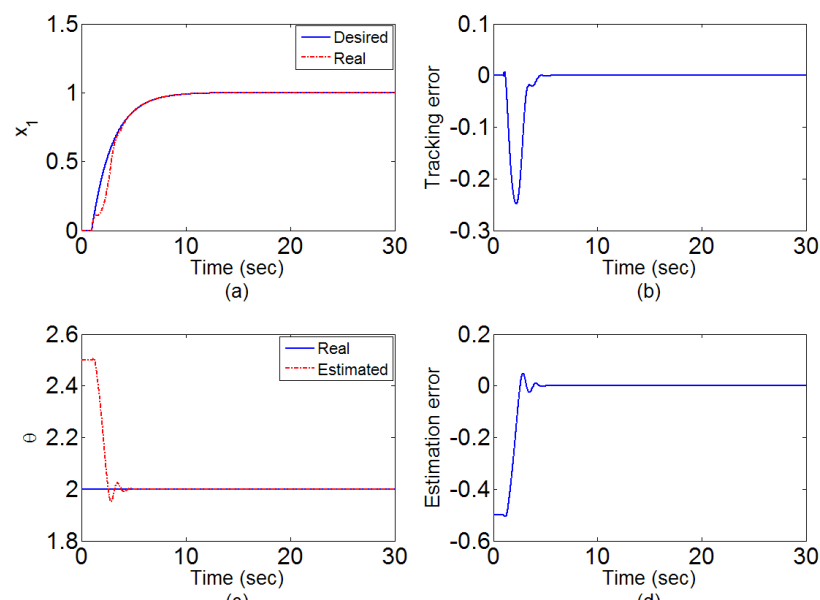

Fig. 1. Simulation results of desired trajectory tracking, tracking error, parameter estimation and estimation error, respectively.

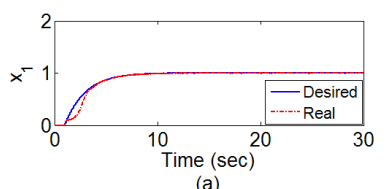

(a)

(c)

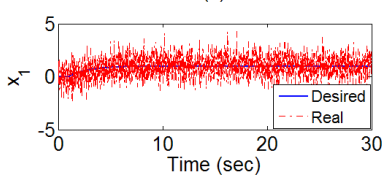

(e)

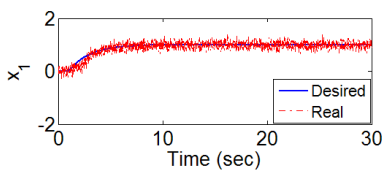

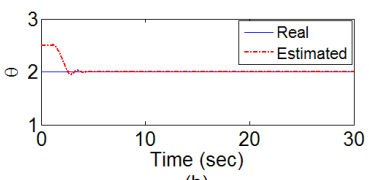

(b)
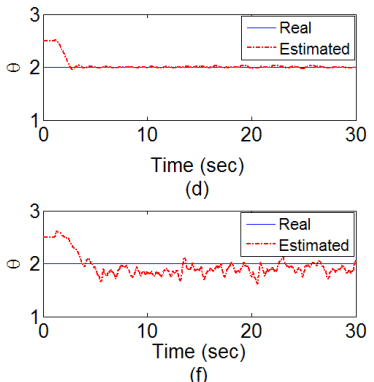

Fig. 2. Simulation results related to the added white gaussian noise of desired trajectory tracking and parameter estimation, respectively.

For the second case, the simulation data are as follows:

$$
\begin{aligned}
x_{1}(0) & =0.35 \\
\gamma & =1 \\
\hat{\theta}(0) & =0.75
\end{aligned}
$$

where the parameter $\theta$ to be estimated is:

$$
\theta(t)=e^{\arctan \left(-\frac{t}{2}\right)}
$$

Despite of the presence of an additive white gaussian noise $w(0, \sigma)$ and the dynamic behavior of the unknown parameter $\theta$, Figure 4 shows a satisfactory performances in term of tracking and estimation, respectively.

\section{CONCLUSION}

This paper describes an approach for developing an indirect adaptive backstepping systematic procedure tracking control design for nonlinearly parameterized class of second-order
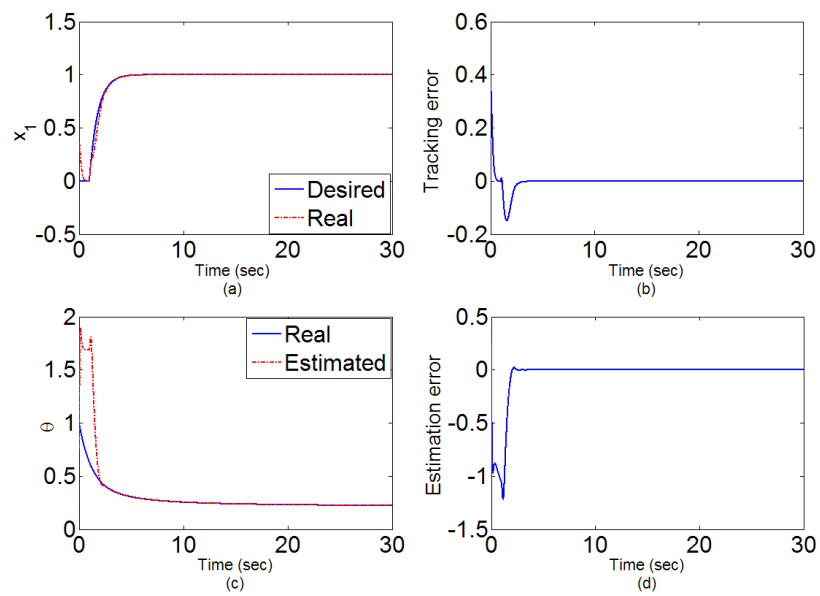

Fig. 3. Simulation results of desired trajectory tracking, tracking error, parameter estimation and estimation error, respectively.
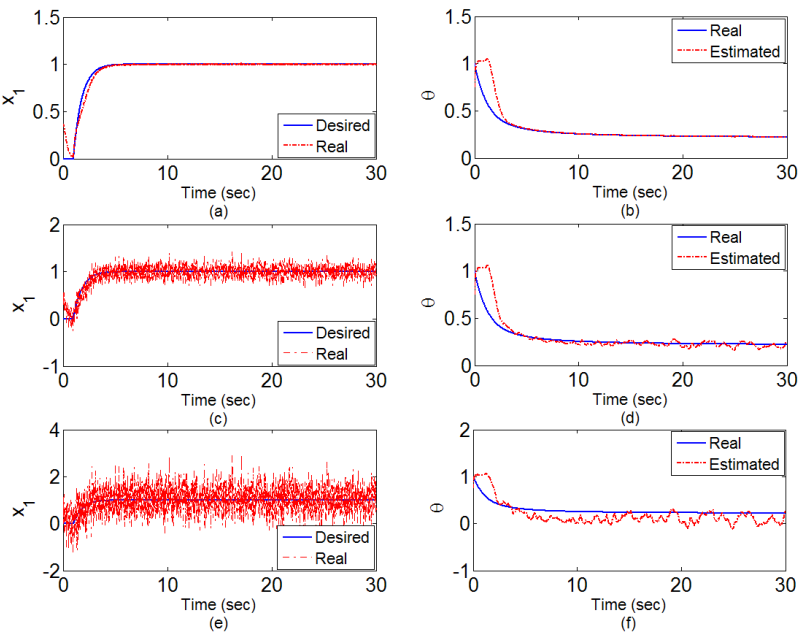

Fig. 4. Simulation results related to the added white gaussian noise of desired trajectory tracking and parameter estimation, respectively.

nonlinear systems without any restriction on the unknown parameter in this case. The choice of the considered class of nonlinear systems is not fortuitous since it is feedback linearizable and verifies the controllability condition $\forall\left(x_{1}, x_{2}\right) \in \mathbb{R}^{2}$ and $\forall f\left(x_{1}, \theta\right) \in \mathbb{R}$. In addition, no internal dynamics is associated to the considered output.

Control design is based on the construction of a Lyapunov positive definite function in two steps where both of the control law and adaptation mechanism are simultaneously synthesized in order to ensure asymptotic Lyapunov stability.

The proposed controller design had been applied to an illustrative numerical example for two cases. In the first case, the adaptation mechanism is related to the estimation of a constant unknown parameter while in the second one, it is related to the estimation of an unknown function.

Both of them, are also studied by adding a centered white gaussian noise to the output $y=x_{1}$ with different variances.

Simulation results show satisfactory performances in term of tracking and estimation, respectively. 
In perspective, we would like to apply developed approach to the longitudinal dynamics for a transportation aircraft.

\section{REFERENCES}

[1] M. Krstic, I. Kanellakopoulos and P. Kokotovic, Nonlinear and Adaptive Control Design, John Wiley and Sons, Inc, 1995.

[2] R. Marino and P. Tomei, Nonlinear Control Design, Prentice Hall International, UK, 1995.

[3] D. Seto, A.M. Annaswamy and J. Baillieul, "Adaptive Control for Nonlinear Systems with a Triangular Structure", IEEE Trans. Automatic Control, 39(1994), 1411-1428.

[4] Z.P. Jiang and L. Praly, "Design of Robust Adaptive Controllers for Nonlinear Systems with Dynamic Uncertainties", Automatica, 34(1998), 825-840.

[5] W. Lin, "Global Robust Stabilization of Minimum-Phase Nonlinear Systems with Uncertainty", Automatica, Vol. 33(1997), 453-462.

[6] J.D. Boscovic, "Stable Adaptive Control of a Class of Continuous-Flow Bioreactor Processes", AIChE J, Vol. 42, no. 1, pp. 176-186, 1996.

[7] J.D. Boscovic, "Stable Adaptive Control of a Class of First-Order Nonlinearly Parameterized Plants", IEEE Transactions on Automatic Control, Vol. 40, pp. 347-350, 1995.

[8] R. Marino and P. Tomei, "Global Adaptive Output Feedback Control of Nonlinear Systems, Part I: Linear Parameterization; Part II: Nonlinear Parameterization", IEEE Transactions on Automatic Control, Vol. 38, pp. 17-48, 1993.

[9] J.D. Boscovic, "Adaptive Control of a Class of Nonlinearly Parameterized Plants", IEEE Transactions on Automatic Control, Vol. 43, no. 7, pp. 930934, July 1998.

[10] Z. Ding, "Adaptive Control of Triangular Systems with Nonlinear Parameterization", IEEE Transactions on Automatic Control, Vol. 46, pp. 1963-1967, 2001.

[11] W. Lin and C. Qian, "Adaptive Regulation of Cascade Systems with Nonlinear Parameterization", IEEE Transactions on Automatic Control, Vol. 38, pp. 33-43, 2002.

[12] A.M. Annaswamy, F.P. Skantze and A. Loh, "Adaptive Control of Continuous Time Systems with Convex Prameterization, Automatica, 34(1998), 33-49.

[13] W. Lin and C. Qian, Adaptive Control of Nonlinearly Parameterized Systems, Proceedings of the $40^{\text {th }}$ IEEE Conference on Decision and Control, Orlando, Florida USA, December 2001.

[14] I. Kanellakopoulos, P. V. Kokotovic and A. S. Morse, "Systematic Design of Adaptive Controllers for Feedback Linearizable Systems", IEEE Transactions on Automatic Control, Vol. 36, No. 11, pp. 12411253, November 1991.

[15] M. Krstic and P. Kokotovic, "Adaptive Nonlinear Design with Controller-Identifier Separation and Swapping", IEEE Transactions on Automatic Control, Vol. 40, pp. 426-440, 1995.

[16] J. Wang and Z. Qu, Robust Adaptive Control of Strict-Feedback Nonlinear Systems with Nonlinear Parameterization, Proceedings of the American Control Conference, Denver, Colorado, June 4-6, 2001.

[17] I.J.-J. Slotine and L. Weiping, Applied Nonlinear Control, Prentice Hall, Upper Saddle River, NJ; 1991. 